\title{
Dumas relève un défi : Les Aventures de John Davys
}

\author{
Vittorio Frigerio \\ Dalhousie University \\ Vittorio.Frigerio@Dal.Ca
}

Rebut: 1 de febrer de 2021

Acceptat: 26 de febrer de 2021

\section{RESUM}

\section{Dumas accepta un repte: Les Aventures de John Davys}

L'article se centra en la novel-la Les Aventures de John Davys, eclipsada per la publicació d'altres cinc obres l'any en què va veure la llum (1840). D'inspiració byroniana, Dumas adopta un estil diferent al de la seva producció anterior i s'emmarca en el gènere de novel-la d'aventures marítimes. Aquesta singularitat va propiciar la hipòtesi de plagi entre alguns crítics. La present contribució revela el controvertit ressò que aquest volum va cobrar en la seva època. Efectua a més, una anàlisi de l'obra i en subratlla, particularment, aquells aspectes que poden mostrar la relació entre l'esmentat text i altres de l'autor per tal de demostrar fins a quin punt l'escriptura de Dumas és múltiple i diversa en la seva forma.

\section{Paraules Clau}

Alexandre Dumas, Revue Britannique, novel-la marítima, novel-la d'aventures, plagi.

\section{RÉSUMÉ}

\section{Dumas relève un défi : Les Aventures de John Davys}

L'article se concentre sur le roman Les Aventures de John Davys, qui a été éclipsé par la publication de cinq autres œuvres l'année de sa parution (1840). D'inspiration byronienne, Dumas adopte un style différent de celui de ses œuvres précédentes et s'inscrit dans le genre du roman d'aventures maritimes. Cette singularité a conduit à l'hypothèse du plagiat chez certains critiques. La présente contribution révèle l'écho controversé que ce volume a eu en son temps. Il procède également à une analyse de l'œuvre, en accordant une 
attention particulière aux caractéristiques qui peuvent montrer la relation entre le texte susmentionné et d'autres de l'auteur, afin de démontrer à quel point l'écriture de Dumas est multiple et diverse dans sa forme.

\section{MOTS CLÉS}

Alexandre Dumas, Revue Britannique, roman maritime, roman d'aventures, plagiat.

\section{RESUMEN}

\section{Dumas acepta un reto: Les Aventures de John Davys}

El artículo se centra en la novela Les Aventures de John Davys, eclipsada por la publicación de otras cinco obras el año en que vio la luz (1840). De inspiración byroniana, Dumas adopta un estilo distinto al de su producción anterior y se enmarca en el género de novela de aventuras marítimas. Dicha singularidad propició la hipótesis de plagio entre algunos críticos. La presente contribución revela el controvertido eco que este volumen cobró en su época. Efectúa además un análisis de la obra atendiendo, particularmente, a aquellos rasgos que pueden mostrar la relación entre el mencionado texto y otros del autor para demostrar hasta qué punto la escritura de Dumas es múltiple y diversa en su forma.

\section{Palabras Clave}

Alexandre Dumas, Revue Britannique, novela marítima, novela de aventuras, plagio.

\section{AbSTRACT}

\section{Dumas: Challenge taken - The Adventures of John Davys}

The article focuses on the novel Les Aventures de John Davys, which was overshadowed by the publication of five other works in the year it was published (1840). Inspired by Byronic inspiration, Dumas adopts a different style from his previous works and falls within the genre of maritime adventure novels. This singularity has led some critics to hypothesise plagiarism. This contribution reveals the controversial echo that this volume had in its time. It also carries out an analysis of the work, paying particular attention to those features that can show the relationship between the aforementioned text and others by the author in order to demonstrate the extent to which Dumas' writing is multiple and diverse in its form.

KEYWORDS

Alexandre Dumas, Revue Britannique, sea novel, adventure novel, plagiarism. 
Dans son édition de décembre 1828, la Revue Britannique - dont la raison d'être est d'offrir un "Choix d'articles traduits des meilleurs écrits périodiques de la Grande-Bretagne, sur la littérature, les beaux-arts, les arts industriels, l'agriculture, la géographie, le commerce, l'économie politique, les finances, la législation, etc., etc. »-publie, sans nom d'auteur, la traduction d'un texte originellement paru dans le New Monthly Magazine, qui débute par une déclaration fort critique envers la catégorie des romanciers :

Ce fut à la fin de 1807 que se passa, dans l'île de Malte, un événement, ou plutôt une série d'événemens du caractère le plus singulier. Je m'étonne que nos faiseurs de romans, qui cherchent à compenser la stérilité de leur imagination en mettant l'histoire au pillage, n'aient pas encore tiré parti des faits dont je fus témoin et dont je conserve le vif souvenir après un intervalle de plus de vingt années. Apparemment qu'ils n'en auront pas eu connaissance.

Douze ans plus tard, Alexandre Dumas, qui a commencé la transition qui le fera passer du rôle de providence des théâtres à celui de premier romancier de France - ne fût-ce qu'en termes de lecteurs -, fait paraître un roman intitulé Les Aventures de John Davys, qu'on aurait été excusé de ne pas trop remarquer en une année, 1840, où son auteur produit également cinq autres ouvrages en des genres divers ${ }^{1}$.

Mais le volume ne passe guère entièrement inaperçu. Cette même Revue Britannique consacre non pas une, mais deux recensions au roman de Dumas. Sans en parler en termes d'un enthousiasme à toute épreuve, elles ne sont pas moins fort élogieuses. La première, publiée après la sortie des deux premiers volumes du roman, juge que

S'il y a beaucoup à critiquer dans ce roman d'aventures, ce serait être ingrat que de chicaner sur quelques détails de composition un auteur qui vient de vous amuser pendant deux volumes et qui vous en promet au moins deux autres tout aussi faciles à faire en apparence, mais que lui seul peut-être peut rendre aussi amusans que les premiers. M. Alexandre Dumas est un narrateur plein de vie, qui marche toujours et qu'on ne surprend guère dans ces longues digressions où se perd si souvent l'intérêt des romans modernes. Il vous entraîne avec lui d'épisodes en épisodes, sans vous laisser le temps de lui demander s'il est toujours dans le vrai ou même dans le vraisemblable. [...] Tout ce qui arrive à John Davys ne constitue pas un drame, mais une suite de scènes, tant bien que

\footnotetext{
${ }^{1}$ Année faste, 1840 voit en effet la parution de plusieurs autres travaux de Dumas : Napoléon, Othon l'archer, Les Stuarts, Maître Adam le Calabrais, et Le Maître d'armes.
} 
mal cousues ensemble, et dont le charme est dans le mouvement et la rapidité de la narration. [...] Encore une fois, si le roman de M. Alex. Dumas n'est pas un livre bien fait, il est plus sûr du succès que tant de romans prétentieux où l'auteur a toutes sortes de succès, excepté celui de se faire lire. ${ }^{2}$

La deuxième critique, traitant des deux derniers volumes, trouve que « les tomes 3 et 4 de l'Odyssée de J. Davys sont à la fois plus amusans encore et mieux composés que les précédens ». Elle souligne l'inspiration byronienne de l'ouvrage (Byron y figure d'ailleurs en tant que personnage), insistant sur un personnage féminin, ce « ravissant prototype byronien, Fatinitza », et estimant que « dans aucun de ses ouvrages, en vers ou en prose, M. Alexandre Dumas n'a dessiné une figure aussi angélique ». Figure dans laquelle le critique voit le plus beau succès du livre, à tel point qu'il juge que « le nom de Fatinitza [doit] désormais évoquer les sentiments les plus tendres et plonger dans la rêverie des poètes le plus prosaïque lecteur de romans $»^{3}$.

Dans un autre article, Joël Cherbuliez, qui, selon Frank Wild Reed, « n'avait pas du tout tendance à faire preuve de clémence envers l'auteur dans ses critiques littéraires ${ }^{4}$, va jusqu'à affirmer que Les Aventures de John Davys sont « l'un des plus jolis romans de notre littérature actuelle ». Il est vrai qu'il préface son jugement d'allusions qui ne cachent guère l'opinion qu'il pouvait avoir de ce que Dumas avait fait jusque-là, affirmant que si « [c]e nouveau roman de M. Alexandre Dumas est très-supérieur aux productions du même genre que cet écrivain a publiées depuis quelque temps $»^{5}, c^{\prime}$ est principalement car « il a renoncé aux niaiseries et aux fanfaronnades, et s'est donné la peine de composer un récit vraisemblable, d'emprunter ses incidens à la vie réelle et sérieuse ». Surtout, Dumas, dans ce roman, aurait montré pour une fois plus de retenue que ses collègues et concurrents ; l'auteur, dit le critique, « a su produire beaucoup d'effet sans avoir recours à ces moyens violens, à ces scènes atroces dont $\mathrm{M}$. Eugène Sue a donné le déplorable exemple si tristement imité par ses nombreux imitateurs ». Ces jugements posés, Cherbuliez identifie une autre source d'inspiration qui aurait permis à Dumas de réussir dans le

\footnotetext{
${ }^{2}$ Chronique littéraire (non signée) de la Revue britannique, janvier et février 1840, p. xxxvi.

${ }^{3}$ Chronique littéraire de la Revue britannique, janvier 1840, p. LVI.

4 "not at all given to the showing of leniency towards its author in his literary criticisms" ( $A$ Bibliography of Alexandre Dumas père, disponible en ligne: http://www.cadytech.com/dumas/ work.php?key=27, consulté le 30 décembre 2020). Les traductions sont de nous.

${ }^{5}$ Jugement conforme à celui du Grand Dictionnaire Larousse, qui considère que « les Aventures de John Davys peuvent être rangées au nombre des meilleures et des plus amusantes productions de notre conteur » (Grand dictionnaire universel du XIXe siècle, Tome 1, part. 4, p. 1055).
} 
domaine, pour lui nouveau, du roman d'aventures maritimes. C'est que son marin anglais aurait « un air de parenté très frappant avec le héros du capitaine Marryat ». Lequel, au sein de la production multiforme du romancier anglais, il ne le spécifie pas. Il ajoute toutefois à cette comparaison une remarque que les appréciateurs de la production dumasienne vont depuis resservir souvent, sous une forme ou sous une autre :

je n'en ferai pas un motif de reproche ; il est permis d'imiter lorsque l'imitation n'est pas trop servile et produit une œuvre qui, sans être tout-à-fait originale, offre du moins une physionomie particulière et se fait lire avec plaisir. Or, c'est justement le caractère du roman de M. Dumas ; la donnée principale est évidemment empruntée à l'auteur anglais, dont les écrits jouissent d'une vogue bien méritée ; mais les détails sont neufs, pleins de verve et de mouvement. ${ }^{6}$

Depuis 1840, l'auteur, encore futur, des Trois Mousquetaires et du Comte de Monte-Cristo, aura l'occasion de fournir bon nombre d'exemples de son habileté à pondre - diront certains - quantité de romans où verve et mouvement abondent. Peu étonnant dès lors que face au fleuve d'encre qui semble couler de la plume de Dumas, ce roman particulier ne tienne pas trop longtemps les devants de la scène. Mais sept ans plus tard, une petite lettre le rappelle aux lecteurs - une fois de plus - de la Revue Britannique. Il s'agit cette fois-ci d'un texte de Thackeray, l'humoriste anglais, intitulé «La continuation d'Ivanhoe. Lettre de Michel-Ange Titmarsh à M. Alexandre Dumas, marquis Davy de la Pailleterie $»^{7}$. Dans cette lettre - qui est la traduction passablement abrégée d'un article paru l'année précédente dans une revue anglaise ${ }^{8}$ Thackeray propose à Dumas, au cas où il serait à court d'idées de personnages attachants pour ses prochaines créations, de redonner vie à des héros dont la carrière littéraire a déjà été couronnée de succès, confiant dans le fait que les lecteurs apprécieront de découvrir la suite de leur destin, après la fin des romans qui ont narré leurs exploits. Pour le convaincre, Thackeray n'hésite pas à avouer être «un partisan décidé du nouveau système dont vous êtes l'inventeur en France. J'aime vos romans en vingt et un volumes » ${ }^{9}$. Le texte original insiste encore davantage, en élargissant ses louanges ironiques aux

\footnotetext{
${ }^{6}$ Joël Cherbuliez, Revue Critique des livres nouveaux, février 1840, p. 38-39.

${ }^{7}$ La Revue Britannique, août 1847, p. 192-194.

8 "Proposals for a continuation of Ivanhoe", Fraser's Magazine, 1846, p. 237-238.

${ }^{9}$ La Revue Britannique, janvier 1847, p. 193. La version anglaise, à peine plus modeste, disait «wenty ».
} 
autres grands maîtres du roman-feuilleton : « Nous vivons des traductions de vos ouvrages ; de ceux de M. Eugène Sue, votre illustre confrère ; de ceux du tragique et mystérieux Soulié, le maître du code criminel ; et de ceux du jeune et bouillant Paul Féval, qui fait concurrence à tous les trois $\gg^{10}$. Mais surtout, la fin de l'article de Thackeray offre une remarque qui va avoir un bel avenir :

J'ai jeté sur le papier quelques indications que je vous soumets, et je vous prie de croire que si vous me faites l'honneur d'en tirer parti, je n'imiterai pas ceux qui vous accusent d'exploiter leurs idées. Je suivrai plutôt l'exemple et le bon goût de la Revue Britannique, qui se contente de se proclamer votre collaboratrice, parce que vous lui avez emprunté un jour, sans mot dire, une nouvelle intitulée Térence le tailleur et la moitié d'un roman: Les Aventures du matelot Davy (sic), dont le nom, au moins, vous appartenait aussi légitimement qu'à elle. ${ }^{11}$

La «moitié d'un roman » (un roman en quatre volumes, même si aux pages très aérées), ce n'est pas peu de chose. On pourrait s'étonner qu'un auteur londonien soit si bien au courant non seulement des détails de la production ancienne de Dumas, mais également de ce qu'avait pu publier bien des années auparavant une revue française, même consacrée à son pays. On aurait raison de le faire. En effet, le passage que nous venons de citer ne figure pas dans l'article original de Thackeray et y a été ajouté par le traducteur, qui avait visiblement lui aussi son opinion bien arrêtée sur la valeur des œuvres de Dumas. Le lecteur français ne le saura pas, et voilà donc que grâce à cette intervention d'un traduttore quelque peu traditore, ce roman en vient déjà à cumuler trois sources d'inspiration (si ce terme neutre est permis) : Marryat, Byron, et surtout un texte imprécisé paru dans la Revue Britannique, qui en aurait fourni «la moitié ».

L'opinion d'un auteur célèbre fait autorité, même lorsqu'elle n'est pas réellement de lui. La remarque faussement attribuée à Thackeray ne tarde pas à être reprise, mais comme il advient souvent dans ces sortes de choses, elle l'est de telle façon que ses citations, au lieu d'éclairer ultérieurement les origines éventuelles du roman de Dumas, finissent par les confondre davantage.

La même année, J.-M. Quérard, dans ses Supercheries littéraires dévoilées, relie la critique de Cherbuliez et le texte humoristique de Thackeray

\footnotetext{
${ }^{10}$ « We live upon translations of your works ; of those of M. Eugène Sue, your illustrious confrère; of those of the tragic and mysterious Soulié, the master of the criminal code; and of the ardent and youthful Paul Féval, who competes with all three." (Art. cit.).

${ }^{11}$ La Revue Britannique, janvier 1847, p. 194. «Térence le tailleur » est une nouvelle insérée en 1842 dans le volume Le Capitaine Aréna.
} 
avec des résultats imprévus. Il rappelle d'abord l'influence présumée de Marryat suggérée par Cherbuliez, et conclut que celui-ci « ne s'est pas trompé dans sa conjecture sur la provenance de ce roman », car « il ne serait pas permis d'émettre aucun doute à ce sujet ${ }^{12}$ depuis la parution du texte de Thackeray - confondant implicitement le roman Les Aventures du matelot Davy, titre incorrect cité dans l'article revu et corrigé de l'écrivain anglais, avec une œuvre du capitaine Marryat, qui aurait de plus soi-disant paru dans la Revue Britannique. La confusion perdure et augmente. Un ouvrage anglais du même genre que celui de Quérard calque ces jugements et affirme aussi que «"John Davy" (sic) est un marin anglais, très proche du héros homonyme du capitaine Marryat. Dans une lettre quelque peu verbeuse de Michael Angelo Titmarsh (Thackeray) au très noble Alexandre Dumas [...] il l'accuse directement de l'avoir adopté (sic) de l'anglais $»^{13}$. Notons en passant que nos recherches ne nous ont pas permis d'identifier un héros portant ce nom dans les romans, très nombreux au demeurant, de l'écrivain anglais ${ }^{14}$, que nous avons abondamment parcourus. La Bibliographie contemporaine de Laporte reprend aussi brièvement le jugement de Cherbuliez et le rapprochement du faux Thackeray, en signalant : «Roman emprunté à une nouvelle de la Revue britannique : les Aventures du matelot Davy (sic), mais dont les détails sont neufs, pleins de verve et de mouvement $»{ }^{15}$. Sauf, on le notera, que le « roman » inspirateur est maintenant devenu une nouvelle ${ }^{16}$. Charles Glinel, dans son

\footnotetext{
${ }^{12}$ J.-M.Quérard, Les Supercheries littéraires dévoilées. Galerie des auteurs apocryphes, supposés, déguisés, plagiaires et des éditeurs infidèles de la littérature française pendant les quatre derniers siècles : Ensemble les industriels littéraires et les lettrés qui se sont anoblis à notre époque, Tome Premier, L'Éditeur, Rue Mazarine, Paris, 1847.

13 "John Davy" is an English sailor, having a close relationship with Captain Marryat's of the same name. In a somewhat prosy letter of Michael Angelo Titmarsh (Thackeray) to the most noble Alexandre Dumas [...] he directly accuses him of having adopted (sic) it from the English" (Olphar Hamst, Handbook for Fictitious Names: Being a Guide to Authors, Chiefly in the Lighter Literatures of the XIX ${ }^{\text {th }}$ Century, Who Have Written Under Assumed Names; and to Literary Forgers, Impostors, Plagiarists, and imitators, John Russel Smith, London, 1868). La coquille fait ici figure, dans les deux langues, de lapsus freudien. Cet auteur n'a visiblement pas lu l'article original de Thackeray, mais brode sur des informations probablement d'origine française.

${ }^{14}$ The Cambridge Bibliography of English Literature (s. la dir. de F.W. Bateson, Volume III, Cambridge University Press, 1969) donne une liste de vingt-quatre romans, et quatre volumes divers.

${ }^{15}$ Antoine Laporte, Bibliographie contemporaine. Histoire littéraire du dix-neuvième siècle, Tome IV, F. Vieweg, Paris, 1887, p. 57.

${ }^{16}$ Le site Gallica, providence des chercheurs, offre une collection de numéros de la Revue Britannique, de 1825 à 1901. Dans aucun de ces numéros ne nous a-t-il été possible de retrouver une nouvelle portant ce titre.
} 
ouvrage bien connu, considère la lettre de Thackeray un " désagrément » pour Dumas, bien relatif par ailleurs, comme « le destinataire de cette lettre se consolait de ces divers déboires dans sa propriété de Monte-Cristo, près de Saint-Germain, où il faisait construire un château merveilleux $\gg^{17}$. Mais ce désagrément va suivre l'auteur à la trace, avec d'autres du même genre, jusque sur les pages qui lui sont consacrées par ce Charles Jean-Baptiste Jacquot, qui, sous le nom d'Eugène de Mirecourt, s'érige en justicier des crimes dumasiens et pontifie, sans trop prendre la peine de prouver quoi que ce soit : "Les Aventures de John Davy (sic) sont empruntées à la Revue Britannique. [...] Le hardi plagiaire prend tout, le plan, le ton, les pages. Il ne se donne pas la peine d'intervertir l'ordre des propositions et de changer dix mots $»^{18}$. On aura le loisir, plus loin, de vérifier, dans ce cas particulier, cette dernière affirmation.

Reed, pour revenir à lui, est le seul à ne pas être pressé de parvenir à des conclusions catégoriques. Il note en effet : «Thackeray a accusé Dumas d'avoir emprunté la moitié de son histoire à la Revue Britannique, mais comme il n'a pas fourni davantage d'indications, la chose demeure plus que passablement incertaine. En 1857, le vrai John Davys était en très bons rapports avec Dumas, et traduisit pour son journal Le Monte-Cristo le Voyage sentimental de Sterne $»^{19}$. De fait, la chose est d'autant plus incertaine que celui qui a porté cette accusation n'était en tout cas pas Thackeray.

\footnotetext{
${ }^{17}$ Charles Glinel, Alexandre Dumas et son æuvre, F. Michaud, Reims, 1884, p. 404-405.

${ }^{18}$ Eugène de Mirecourt, Dumas père, Librairie des contemporains, Paris, 1869, p. 24. Encore de nos jours la confusion ne cesse de se manifester et de varier davantage. Ainsi peut-on trouver en ligne l'abrégé suivant : «Pour l'anecdote, il semblerait d'après Franck Wild Reed, que le romancier anglais Thackeray (Mémoires de Barry Lyndon, La foire aux vanités...) ait accusé Dumas de lui avoir "emprunté" une partie de l'histoire... » (http://www.dumaspere.com/pages/dictionnaire/ aventures_john_davys.html). Le Dictionnaire Dumas de Réginald Hamel et Pierrette Méthé, sans indiquer source aucune, affirme que le roman serait « une transposition d'un ouvrage de langue anglaise » (Guérin, Montréal, 1990, p. 34).

${ }^{19}$ « Thackeray accused Dumas of borrowing half his story from the "Revue Britannique," but as he gave no further indication, there seems still to be more than a little uncertainty about this. In 1857 the actual John Davys was on very friendly terms with Dumas, and translated for the pages of "Le Monte-Cristo" Sterne's "Sentimental Journey » (Op. cit.). Dumas présente en effet ainsi son ami dans les pages de son journal : « Mais vous ne connaissez pas mon ami John Davys. Mon ami John Davys, dont j'ai autrefois donné le nom à un héros de mes romans, est aujourd'hui un homme de trente-cinq à trente-huit ans, grand, sec, grave, avec un sourire d'une finesse extrême. C'est un Anglais élevé en France, parlant français comme vous et moi, et très versé dans la littérature des deux pays » («Causerie avec mes lecteurs », Le Monte-Cristo, jeudi 17 décembre 1857).
} 


\section{Dumas : un Marryat français?}

Pour compliquer, ou pour éclairer, ultérieurement l'affaire - cela reste à voir - un article particulièrement virulent paru dans The Eclectic Magazine en 1856 , dans lequel on déclare que «pas la vingtième partie des ouvrages portant sur leur page de couverture les mots par Alexandre Dumas n'ont été écrits par cet individu $»^{20}$, s'étonne hautement qu'un écrivain français ait pu représenter avec une telle précision la vie à bord d'un vaisseau de sa majesté. La raison du mystère est toute trouvée : Dumas aurait tout simplement ajouté les œuvres du capitaine Marryat à la liste de celles dans lesquelles il s'est librement servi dans sa longue, et peu illustre, carrière : " La bataille, l'orage et le naufrage sont bien représentés [...] en des termes nautiques. [...] Ne trouve-t-on pas cette scène de bataille dans Peter Simple, cet orage dans Newton Foster ? $»^{21}$. Il n'y a pas à en douter, les scènes de batailles navales et d'orages n'étant pas trop rares dans les romans maritimes. L'auteur de l'article n'offre malheureusement pas davantage de détails pour étayer ses affirmations, mais renvoie son lecteur éventuellement curieux aux ouvrages de Quérard et de Mirecourt, en plus qu'à l'Alexandre Dumas dévoilé de "Chassagnac $»^{22}$ (sic) - références sur lesquelles on sait à quoi s'en tenir, qu'il qualifie d' « autorités » sur le sujet ${ }^{23}$. Ces jugements sont assez largement partagés outre-Manche, quoiqu'avec

\footnotetext{
20 "not one-twentieth of the works bearing the words par Alexandre Dumas on their title-pages were written by that individual" ("The Literary Leviathan", The Eclectic Magazine, August 1856, pp. 491-96). Tous mes remerciements à Julia Wright pour m'avoir signalé ce texte.

21 "The battle, storm and wreck, are [...] ably and nautically depicted. [...] Is not this battle-piece in Peter Simple, this storm in Newton Foster?" (Ibid).

${ }^{22}$ Il s'agit évidemment de l'ouvrage de Granier de Cassagnac, paru sans nom d'auteur en 1847.

${ }^{23}$ La critique britannique contemporaine se montre en général peu compréhensive envers Dumas, se plaisant souvent à le brocarder non seulement pour l'immoralité de ses feuilletons - défaut qu'il partage avec tous les autres romanciers français -, mais pour son titre et ses origines. Il n'est ainsi pas bien difficile de trouver des jugements comme celui publié dans le Fraser's Magazine de mai 1846 (« On a late French Trial », non signé, p. 621-630), où après la condamnation d'usage des «productions monstrueuses de l'imagination malade de ces écrivains célèbres » (« the monstrous productions of those famous writers' diseased imagination » [621]), on note: "Ceux qui savent l'histoire de cet auteur, qu'il a lui-même contée, et le fait que son père était un général mulâtre célèbre dans les guerres révolutionnaires, seront surpris d'apprendre que son fils est un marquis ; car bien que nous ayons entendu parler du Marquis de Marmalade et du Duc D'Alicompaine à Hayti (sic), nous ne savions pas, jusqu'ici, que les Français avaient reconnu une aristocratie nègre. ( « Those who know, from the author' own writings, his history, and that his father was a mulatto general famous in the revolutionary wars, will be surprised to hear that the son is a marquis; for though we have heard of the Marquis of Marmalade and the Duke of Alicompaine in Hayti [sic], we did not know, until now, that the French had recognized a negro aristocracy » [628]). Dans le même article, Dumas est qualifié d' « infortuné, pauvre mécréant » (« miserable, poor heathen » [628]).
} 
des variations sensibles sur le pourcentage de son œuvre dont Dumas serait réellement responsable. Une autre critique parue dans le Harvard Magazine affirme - reproduisant les arguments de son prédécesseur - qu' «il n'a pas écrit lui-même la dixième partie des ouvrages que l'on présente comme siens » et que son dernier roman «a été pour la plupart approprié des œuvres du capitaine Marryat $»^{24}$. De fait, même les critiques les plus positifs mélangent leurs louanges à des caveat significatifs : " Il y a un roman maritime par Alexandre Dumas où il a réussi à notre avis mieux que dans tous ses autres travaux [...]. [I]l y fait montre d'une connaissance des affaires maritimes anglaises à laquelle on ne se serait jamais attendu de Dumas, quand on pense à l'ignorance complète des mœurs anglaises qu'il a montrée dans son drame Kean. Depuis lors, Dumas [...] a dû "plancher sur Marryat et Cooper", et certainement avec grand profit $»^{25}$.

Il est effectivement très loin d'être impossible que Dumas ait eu entre ses mains les romans de Marryat, qui jouissaient en France, dans les années précédant la parution des Aventures de John Davys, d'un engouement certain. « Nommer pour auteur le capitaine Marryat, c'est annoncer un succès, car tout le monde a lu Jacob Fidèle, Pierre Simple, romans bien écrits et amusans », récite une annonce dans Le Charivari du 5 mai 1837. L'intention publicitaire évidente ne devrait pas minimiser la réalité de la vaste diffusion de l'auteur. Au mois de décembre le libraire Gustave Barba publie une édition populaire des Euvres complètes du capitaine, déjà largement répandues à travers l'édition de Charles Gosselin. On se dispute l'auteur dans des traductions rivales, et si Dumas pioche dans ses romans, il n'est au moins pas le seul à le faire : le 23 juillet 1840 débute au Théâtre-Français la pièce Japhet, ou La recherche d'un père, de Scribe et Émile Vanderburk, basée sur le roman homonyme de Marryat, que Gosselin avait publié trois ans auparavant. La reprise en vrac de pages entières de Marryat dont on accuse librement le romancier français, présentant la chose comme acquise, demeure toutefois plus difficile à prouver.

\footnotetext{
24 "not one tenth of the books which purport to be his were written by himself. [...] Dumas's novel, "The Adventures of John Davys," is mostly appropriated from Captain Marryat" (R. Ballow, "Literary Frauds", Harvard Magazine, Vol. VIII, 1862, p. 26).

25 "There is a sea novel, by Alexandre Dumas, in which we think he has succeeded better than in any of his other works. [...] there is an acquaintance with the naval affairs of England displayed, which we would certainly never have expected from Dumas, when we call to mind the utter ignorance of English matters manifested in his drama of Kean. Dumas must, since then, [...] have been "reading up his Marryat and Cooper", and certainly with great success" (Henry Vincent Falkland, "Literature in France", Hood's Magazine, Vol. 10, July to December 1848, p. 174-175).
} 
Pour juger du bien-fondé de ces accusations, nous avons vérifié dans treize ouvrages de Marryat - dont ses romans les plus célèbres ${ }^{26}$ - la présence d'une série de mots-clés glanés dans le roman de Dumas, et nous avons essayé d'y retrouver des phrases entières ou des expressions tirées des passages descriptifs du roman de Dumas, en nous concentrant surtout sur les scènes nautiques ou de bataille. Il y a bien une frégate nommée Trident, nom du navire de John Davys, dans M. le Midshipman Aisé, mais elle est russe et à part le nom, commun en marine, rien ne rapproche les deux bateaux. Dans Frank Mildmay et dans Le pauvre Jack il y a des scènes de punition à bord d'un navire, où l'on fait plus ou moins largement usage du chat à neuf queues. Une scène du même ordre se retrouve chez Dumas, mais sans que les deux aient quoi que ce soit en commun, dans le ton, le vocabulaire, la longueur ou leur rôle dans l'intrigue. Il ne nous a pas été possible de retrouver dans cet échantillon, incomplet mais important, des romans de Marryat, la moindre ressemblance évidente avec des scènes du roman de Dumas. Certainement pas d'emprunts systématiques et grossiers, tels ceux que la critique du temps dénonce.

En dépit de ces résultats, ou de cette absence de résultats, il n'est évidemment pas plus justifiable d'affirmer l'inexistence complète d'une influence de Marryat sur Dumas qu'il ne l'est d'affirmer arbitrairement un plagiat quasi-total. Les influences peuvent se faire sentir bien autrement que simplement à travers la reprise mécanique de passages, de situations, de personnages ou d'intrigues. Il n'est pas à exclure, ainsi que le soupçonnait le critique du Hood's Magazine, que la familiarité, pouvant paraître surprenante, de Dumas avec la terminologie nautique et les détails de la vie sur les bateaux de la marine anglaise ne soient dus à sa fréquentation des œuvres du romancier britannique. Mais d'autres sources demeurent tout aussi crédibles : Cooper, bien sûr, mais pourquoi pas Eugène Sue, l'inventeur français du roman maritime, ou quelqu' un de ses épigones de l'époque, bien oubliés maintenant ?

Ce qui saute aux yeux, cependant, sont aussi certaines différences significatives entre Dumas et Marryat. Le héros éponyme des Aventures de John Davys est notamment aidé dans son plan pour se venger des cruelles vexations du lieutenant Burke, par un juif, qui lui fournit des habits civils et lui réserve le passage sur un navire devant l'amener en Italie, après le duel dans lequel il tue son adversaire. L'œuvre de Marryat déborde de personnages de juifs marchands d'habits, prêteurs sur gages ou usuriers, présents dans chaque

\footnotetext{
${ }^{26}$ Pour les besoins de cette recherche nous avons eu recours à l'abondante collection du Centre Sablé de l'Université de Toronto, dont bon nombre d'ouvrages scannés sont disponibles librement en ligne. La liste des romans dans lesquels nous avons effectué des vérifications, dans leur édition française d'époque, figure à la fin de cet article.
} 
port et toujours prêts à arnaquer les marins de passage, tous invariablement présentés en des termes violemment antisémites guère rares dans la littérature anglaise de l'époque. Dumas, dans son portrait de ce personnage, auxiliaire utile du héros, demeure fidèle à sa philosophie universaliste, qui, - tout en n'hésitant pas à attribuer des caractères spécifiques aux nations, sur la lignée de Montesquieu - rejette les condamnations en bloc, et insiste souvent et volontiers sur les aspects positifs, vecteurs de progrès et espérances d'amélioration, qui font que l'humanité entière peut avancer ensemble vers un futur de paix et d'égalité, même si à des vitesses variables ${ }^{27}$.

De plus, les personnages positifs, quoique britanniques, doivent du moins en partie leur valeur à l'influence de la culture française. La mère du héros, orpheline, fille d'un baron ayant dû s'exiler en France car il s'était rangé du côté du prétendant, Charles Stuart, a été élevée par Mlle de Villevieille, une Française, et ses qualités morales, sur lesquelles la narration insiste, sont directement liées à cette éducation. Aussi, le capitaine du Trident, Stanbow, « avait conservé la tradition des formes élégantes et des manières polies, et s'était même fortifié dans le culte de cette tradition par un séjour de trois ou quatre années en France $\aleph^{28}$. Même les souvenirs du père de John, lui-même officier de marine, qui a perdu une jambe dans une bataille contre les Français, présentent une admiration pour l'ennemi battu paraissant pour le moins suspecte de la part d'un personnage anglais :

Puis vint l'histoire de ses onze combats, et du dernier, le plus terrible de tous, où, une cuisse emportée, il s'était relevé sur le pont pour battre des mains en voyant s'abîmer un vaisseau dont l'équipage tout entier avait mieux aimé périr que de se rendre, et s'était enfoncé dans la mer, son pavillon cloué à son grand mât, et aux cris de : «Vive la France ! vive la République ! (JD 9)

Le roman contient également diverses représentations critiques ou ironiques de la société anglaise, ainsi que des rappels épisodiques mais persistants de la supériorité des mœurs ou des vertus françaises, qu'on s'imagine mal figurant dans un roman de Marryat et qui n'apparaissent pas comme étant simplement plaquées par-dessus un texte qui aurait soi-disant été recopié. Ainsi, la cuisine d'outre-Manche, sujet habituel des lazzis français

\footnotetext{
${ }^{27}$ On se souvient du long discours figurant dans le roman La San Felice, où Dumas prend position contre les discriminations envers les juifs (La San Felice, Gallimard, Paris, 1996, p. 504-507).

${ }^{28}$ Aventures de John Davys, Le Vasseur, Paris, s.d., p. 19 (dorénavant indiqué par JD dans le texte).
} 
dirigés contre l'Angleterre, apparaît évidemment en première place - ce qui n'étonne guère de la part du futur auteur du Dictionnaire de cuisine :

Quiconque a mis le pied à bord d'un bâtiment de guerre anglais sait ce que c'est que le dîner d'un midshipman. Un morceau de bœuf à demi rôti, des pommes de terre cuites à l'eau et revêtues de leur robe grise, une liqueur noirâtre baptisée du nom usurpé de porter, le tout dressé sur une table boiteuse, couverte du torchon qui sert à la fois de nappe et de serviette, et qu'on renouvelle tous les huit jours, forment l'ordinaire des Howes futurs et des Nelsons à venir. Heureusement, je sortais du collège et mon apprentissage était fait. (JD 18)

Un brasseur, personnage comique, met sur le compte de «l'imprudence qu'il avait faite de boire deux doigts de vin, liqueur pernicieuse s'il en fut jamais », « l'indisposition dont il venait d'être victime et qui n'était aucunement due à l'intempérance », tout en déplorant les habitudes culinaires de la mère du héros, car on «sai[t] bien, que c'est l'habitude des Françaises de boire de l'eau et de manger des sauterelles » (JD 10). Pour un roman soi-disant calqué sur un modèle anglais, ces nombreux passages peuvent surprendre.

\section{Un « roman » qui n'en est pas un}

Ces points établis, voyons brièvement l'intrigue des Aventures de John Davys pour pouvoir en analyser plus posément le contenu.

L'histoire, narrée à la première personne (ce qui est assez inhabituel pour l'auteur), commence avant même la naissance du personnage, racontant d'abord la rencontre de ses parents. Sa jeunesse fait l'objet de la première partie, comprenant les années passées dans un collège. À cela suivent l'enrôlement dans la marine et les expériences du héros sur le navire Trident. La rivalité avec le lieutenant Burke, supérieur tyrannique, coupable de la mort d'un innocent, le mène à le défier en duel. L'ayant tué, le héros est obligé de se sauver. Le navire qui l'emporte, sur lequel il fait la connaissance d'un Grec du nom d'Apostolidi, avec qui il se lie d'amitié, est abordé par des pirates. Après la bataille, ayant sauvé la vie au fils du chef pirate, qu'il avait lui-même blessé lors du combat, John Davys est accueilli dans leur île, où il tombe amoureux de la fille du chef, la belle Fatinitza. Voulant l'épouser, en dépit de leurs différences de rang et de nationalité, il décide de rentrer en Angleterre pour obtenir l'autorisation de ses parents. Il l'obtient et est exonéré par le tribunal pour la mort de Burke, mais le voyage se prolonge bien au-delà de ses attentes. Lors du retour, en traversant l'Albanie, il fait la rencontre du tristement célèbre 
Ali-Pacha. Arrivé enfin sur l'île grecque où sa fiancée devrait l'attendre, il découvre que, selon les anciennes traditions de ces descendants des Spartiates, elle, et l'enfant qu'elle venait d'avoir de lui, ont été tués par le père et le frère pour venger l'honneur familial.

Comme on le voit, le roman porte les traces d'au moins trois genres différents : le roman d'apprentissage, le roman maritime, et le roman d'aventures et voyages. Ainsi qu'on l'a noté, il est construit essentiellement autour d'une série d'anecdotes qui se suivent, sans charpente narrative particulière, le long du « doux voyage de [1]a jeunesse » et « sur l'âpre et rude chemin de l'avenir » (JD 18). S'il s'avère difficile de retrouver dans ces pages les cas patents de plagiat dont on a accusé l' auteur, il est toutefois bien plus aisé d'y identifier des séries de thématiques, de situations et de réflexions typiques de Dumas, qui se développeront davantage dans ses productions ultérieures jusqu'à devenir, dans certains cas, l'équivalent de marques de fabrique. On peut facilement en passer certaines en revue. En premier lieu vient l'éducation à la liberté et au respect de l'autre, véhiculée à travers la narration d'une expérience d'enfance. Le héros possédant une volière, sa mère le sensibilise à la souffrance des oiseaux, dans une allégorie transparente de la condition humaine :

Elle m'expliqua que c'était injuste de profiter de la détresse du faible pour le réduire en esclavage ; elle me montra les oiseaux, aux premiers bourgeons qui reparurent, essayant de passer à travers le treillage pour se répandre au milieu de cette nature qui revenait à la vie, et ensanglantant leurs petites têtes aux barreaux de fil de fer qui les retenaient captifs. Pendant une nuit, un d'eux mourut ; ma mère me dit que c'était le chagrin de ne pas être libre. Le même jour, j'ouvris la cage, et tous mes prisonniers s'envolèrent en chantant dans le parc. (JD 12)

Ensuite vient l'éducation au courage. Chargé d'une mission dangereuse par le capitaine, John sent que « du moment que ma vie courait quelque chance, du moment qu'il y avait lutte enfin, il pouvait y avoir victoire, et la victoire justifie tout : c'est le talisman qui change le plomb en or » (JD 20). En troisième lieu, la prise de conscience du poids de l'injustice dans la vie sociale : « la loi n'est pas toujours le droit » (JD 29). Face à la manifestation de « la violence, légale peut-être, mais injuste » (JD 30), le héros choisit d'exercer la « vengeance » (JD 24), qui est la concrétisation de la justice individuelle, placée au-dessus des conventions sociales. Celles-ci se révèlent fausses de plus d'une manière, et les personnages positifs du roman sont inévitablement dépeints comme empreints de vertus morales qui leur accordent une noblesse d'âme bien supérieure à toute noblesse de naissance. 
Ainsi, David, homme du peuple, victime innocente de la cruauté du lieutenant Burke, avait « une dignité qui semblait appartenir à un autre rang que celui qu'il avait reçu du hasard » (JD 31), et les pirates grecs chez qui le héros trouve un refuge adoptent un comportement bien au-dessus de leur condition apparente : « il y avait même dans ces deux hommes une instruction et une délicatesse qui semblaient si mal s'accorder avec leur état, que plusieurs fois je m'étais étonné de cette anomalie » (JD 71). Le héros lui-même, de naissance aristocratique, devient « un proscrit » (JD 72), non sans évoquer d'illustres prédécesseurs comme Hernani et bouclant ainsi la boucle entre le haut et le bas de l'échelle sociale. Autre élément important, qui manque rarement dans les romans dumasiens, la découverte de l'amitié sincère et inconditionnelle, qui se manifeste souvent entre personnes très différentes. Ici, la rencontre entre John et Apostoli, le patriote grec mourant de phtisie, remplit plus qu'adéquatement cette fonction. À travers ce personnage, Dumas prête une dimension héroïque aux pirates du roman, « aigles de mer aux ailes rapides, qui fondent sur leur proie, puis se retirent dans des nids trop élevés pour que le lourd despotisme turc ose les y poursuivre », représentants d'un «peuple opprimé ». Et les mots de l'ami du héros anticipent bien d'autres créations dumasiennes ultérieures qui toucheront à ce thème (El Salteador, Pascal Bruno) : "L'Espagne a ses guérillas, la Calabre ses brigands, le Magne ses Klephtes, l'Archipel ses pirates. Vienne le jour de la liberté, et tous redeviendront des citoyens » (JD 62).

On ne peut, pour terminer cette analyse trop rapide, oublier de mentionner la dialectique entre Providence, destin, fatalité et volonté - une constante du discours dumasien - qui sous-tend la partie du roman narrant l'amour impossible entre John et Fatinitza ${ }^{29}$.

Les Aventures de John Davys sont ainsi clairement un roman de Dumas, portant en bonne vue les stigmates reconnaissables qui caractérisent l'essentiel de sa production. Mais justement, un roman dumasien, est-ce simplement un roman original de Dumas?

\footnotetext{
${ }^{29}$ « [E]ntre deux personnes qui se quittent, se place aussitôt une divinité terrible, qui n'est plus la providence, mais le hasard » (JD 84) ; « [T]out ce qu'il est humainement possible de faire pour revenir à moi tu le feras ; mais le destin ne peut-il pas être plus fort que la volonté ? » (JD 97). Pour une analyse moins allusive de ces diverses caractéristiques de l'esthétique dumasienne, nous renvoyons à nos livres Les fils de Monte-Cristo. Idéologie du héros de roman populaire (Presses de l'Université de Limoges [PULIM] Limoges, 2002), et Dumas l'irrégulier (Presses de l'Université de Limoges (PULIM), Limoges, 2011).
} 


\section{De l'art du recyclage}

La structure du roman, construit comme on l'a vu par une succession de tableaux, permet l'inclusion de scènes susceptibles d'en prolonger en principe indéfiniment la longueur. Le voyage du héros en Angleterre et retour (marqué par une rapidité hallucinante qui anticipe les déplacements du Comte de Monte-Cristo et les chevauchées furieuses des mousquetaires) est aussi interrompu par des arrêts. Un de ceux-ci permet à l'auteur de narrer une partie de la sanglante aventure d'Ali-Pacha, le tyran de Janina dont la figure réapparaîtra dans l'histoire d'Haydée, l'amoureuse d'Edmond Dantès. Or, il se trouve que c'est bien entre 1839 et 1840 que paraissent les volumes 7 et 8 des Crimes célèbres, dans lesquels, sous la signature de Félicien Mallefille ${ }^{30}$, est présenté le parcours de ce despote oriental. Le texte de Mallefille est composé, nous dit-il, «d'après les savantes recherches de M. de Pouqueville »" C'est également à l' Histoire de la Grèce de ce même auteur que renvoie Dumas dans son roman " pour connaître la vérité de tous ces détails » (JD 91), indiquant en plus volume et chapitre. Si on peut estimer sans peur de se tromper que Dumas a utilisé le résumé de Mallefille pour base, il ne l'a ni simplement repris, ni copié. Peu de passages ont des airs de famille évidents, et même ceux-là ont fait l'objet de modifications qui en affectent sensiblement le fond ${ }^{32}$. Dumas récupère également un personnage secondaire, la jeune fille Vasiliki, dont l'anecdote est rendue très brièvement par Mallefille (p. 81-82), pour en faire une figure plus marquante, qui croise par deux fois, en des moments clé, le chemin du héros.

D'autres histoires, en quelque sorte enchâssées, sont fournies par la mythologie grecque, citée par Apostoli, dont notamment la légende de Polycrate, empruntée d'Hérodote ; mais ces souvenirs de l'antique ne sont pas dénués de buts comparatifs avec le présent et ne peuvent donc pas être considérés des insertions gratuites simplement destinées à rallonger le feuilleton.

\footnotetext{
${ }^{30}$ Alexandre Dumas, Arnould, Fournier, Fiorentino et Mallefille, «Ali-Pacha », Crimes célèbres, Administration de Librairie, Paris, Tome 7, 1840, p. 307-334 et Tome 8, 1840, p. 3-146.

${ }^{31} \mathrm{Ibid}$, Vol. 7, p. 313.

${ }^{32}$ Un seul exemple, le discours d'Ali-Pacha essayant de persuader à ses soldats chrétiens de massacrer les habitants de Cardiki, qu'il a attirés dans un guet-apens. Mallefille écrit : " C'est à vous, braves Latins, s'écrie-t-il, que je confie maintenant le soin d'exterminer les ennemis de mon nom. Vengez-moi, et je reconnaîtrai ce service par les plus grandes récompenses ! (p. 44); Dumas reformule ainsi, donnant au texte une toute autre dimension : «C'est à vous, braves Latins, s'écria Ali, que j'accorde l'honneur d'exterminer les ennemis de votre religion ; frappez au nom de la croix, frappez au nom du Christ ; tuez ! tuez ! » (JD p. 91).
} 
Une de ces histoires enchâssées, particulièrement tragique, ressort avec force. Le bâtiment de John Davys arrive à Malte le lendemain de l'exécution d'un groupe de soldats d'origines diverses (Albanais, Esclavons, Grecs, Smyrniotes) appartenant au régiment de Frohberg, qui s'étaient insurgés contre l'autorité britannique. Le héros introduit cette mini-narration presque en s'excusant de dévier du parcours de sa propre vie : " un régiment tout entier s'était révolté, et venait d'être détruit par la corde, le fer et le feu, jusqu'au dernier homme, et cela avec des circonstances si particulières, que ce récit, je l'espère, si en dehors qu'il soit de mes propres aventures, ne sera pas sans intérêt pour le lecteur » (JD 33). L'anecdote est fort simple : indignés par la discipline inhumaine qui leur est imposée par leurs instructeurs allemands, qu'ils ressentent comme une insulte, ces soldats, « les hommes les plus libres du monde, après les Arabes du grand désert », « se ru[èrent] sur les officiers, dont la sévérité avait si longtemps tenté leur vengeance [et] les égorgèrent comme des lions eussent fait de gladiateurs dans un cirque » (JD 33). Bon nombre de ces révoltés sont massacrés par les troupes anglaises dans des scènes d'une férocité révoltante. Sept d'entre eux se barricadent dans le fort Ricasoli (que Dumas écrit « Ricazoli »), où ils résistent aux assauts des troupes anglaises pendant une semaine. N'ayant plus ni eau ni vivres, ils font sauter le magasin à poudres du fort. On les croit morts dans cette explosion, mais en réalité ils se sont évadés à travers un passage creusé sous les murs du fort. Réfugiés dans une caverne près de la mer, affaiblis par le manque de nourriture, ils sont découverts quelques jours plus tard, faits prisonniers et rapidement exécutés

L'anecdote parait, de prime abord, n'avoir aucun rapport avec l'histoire du héros. Elle est parfaitement autonome par rapport à l'intrigue du roman, et cela à un tel point que déjà en février 1840, la même année de la parution des Aventures de John Davys, elle est republiée séparément dans le Journal des journaux ${ }^{33}$, quoique sous le titre fautif « Exécution d'un régiment d'Albanais

\footnotetext{
${ }^{33}$ Publication qui se définit « Revue de la presse non politique ou recueil de feuilletons, contes, anecdotes, épisodes et articles remarquables, tant inédits qu'extraits de la presse contemporaine ». L'histoire figure aux pages 31-39. Une première version fictionnelle de cette histoire peut se trouver dans l'ouvrage anonyme Mémoires d'une contemporaine. La contemporaine à Malte et à Alger (Paris, Moutardier, 1833, Chapitre IX, p. 124). Il s'agit d'un compte rendu très dilué des événements, qui suit également dans l'essentiel la version de la Revue Britannique, tout en ajoutant certains détails qui n'y figurent pas - comme le fait que les révoltés avaient changé le nom de leur régiment en « les Indépendants ». Une reconstruction historique de la révolte, bien mieux documentée que les autres, se trouve dans l'article de H. De Schaller, «Le Régiment de Watteville au service de l'Angleterre » (Revue Historique Vaudoise, no. 12, décembre 1894, p. 353-356). On y apprend notamment que le régiment prenait son nom du comte Tulliers de Frohberg ( les ruines de Frohberg, en français Montjoie, [...] sont situées en Franche-Comté »).
} 
à Naples ». Or, ce passage est effectivement repris d'un article publié dans la Revue Britannique du mois de décembre 1828 (p. 219-233), intitulé « La révolte du régiment de Frohberg », ayant paru originellement dans le New Monthly Magazine ${ }^{34}$.

Dumas a-t-il copié cette histoire ? Il l'a sans doute récupérée, suivant pas à pas son développement sans modifier l'ordre des événements. Mais il l'a également enrichie considérablement, développant certaines parties, ajoutant des détails dramatiques inédits, multipliant les commentaires sur la nature indépendante des rebelles, sur leur incapacité à se plier à une discipline que leurs caractères nationaux ne pouvaient que percevoir comme odieuse ${ }^{35}$. Ce passage, prétendument sans rapport aucun avec l'histoire du héros, se transforme ainsi en une préfiguration de la confrontation entre John Davys et le lieutenant Burke - entre une nature droite, qui obéit à des impératifs éthiques universels, et une autorité injuste, abusive, sadique, qui humilie et mortifie les inférieurs et les innocents. Loin d'être simplement une façon d'allonger de quelques pages un roman "mal ficelé », qui avancerait à l'aveuglette d'une anecdote à l'autre, sans savoir où il va, ce passage anticipe un moment important de l'intrigue et - à travers les figures des sept Grecs dont l'astuce arrive un temps à mystifier leurs maîtres anglais - présage également l'entrée en scène des pirates grecs, descendants des fiers Spartiates, qui luttent pour libérer leur pays du joug ottoman. Si on a accusé Dumas d'être un entrepreneur en littérature, ce qui n'est pas faux, on doit pouvoir partager, après une analyse attentive, le jugement exprimé par Le Charivari (5 novembre 1844) : « M. Alex. Dumas est comme M. Rothschild, il fait valoir l'argent des autres par le sien, avec cette différence qu'il n'emprunte pas à tout le monde. Ne lui prête pas qui veut : il choisit, et choisit bien ». Le malheureux destin du régiment de Frohberg avait de quoi attirer le romancier. Et son écho peut s'entendre à plusieurs moments dans son œuvre, que ce soit dans la terrible scène de la mort des compagnons de Jéhu, dans le roman éponyme - qui a plus qu'un air de famille avec ce passage - ou dans ses Mémoires, là où il évoque (sans

\footnotetext{
${ }^{34}$ La nouvelle de la révolte de ce régiment avait également eu des échos dans la presse française. Le Journal de l'Empire la donne dans son édition du 12 juin 1807, offrant un résumé de l'événement qui concorde en tous points avec le texte de la Revue Britannique.

${ }^{35}$ Pour vérifier le retravail accompli par Dumas sur le texte original, nous avons recopié l'extrait du roman et l'article de la Revue Britannique, pour les comparer ensuite en utilisant la fonction idoine du logiciel Word. Le document qui en résulte montre de manière visuellement frappante l'étendue de la refonte opérée par le romancier. Aucune phrase ne demeure intouchée. Quoi qu'en ait dit Mirecourt, Dumas fait beaucoup plus qu'intervertir l'ordre des propositions et changer dix mots. Il modifie systématiquement le texte de départ pour se l'approprier et intensifie l'effet dramatique de l'anecdote, ajoutant nombre de remarques de son cru.
} 
cependant y avoir assisté en personne) la lutte des combattants du cloître SaintMerry lors de l'insurrection de 1832 ; ou même encore, sur un ton bien plus léger, dans le siège de La Rochelle des Trois mousquetaires. Dans tous les cas, il s'agit bien de la résistance héroïque et désespérée d'un petit groupe de personnages courageux et astucieux face à un ennemi prépondérant. Ce qui est une thématique on ne peut plus dumasienne.

On nous pardonnera de croire que Dumas ait dû se sentir mis au défi par l'incipit de l'article, suintant le sarcasme, où l'auteur s'étonnait « que nos faiseurs de romans, qui cherchent à compenser la stérilité de leur imagination en mettant l'histoire au pillage, n'aient pas encore tiré parti des faits dont [il] fu[t] témoin ». Ces mots représentaient une gageure réellement impossible à ignorer pour un auteur faisant déjà régulièrement l'objet de telles accusations.

Malgré ce que le faux Thackeray de la Revue Britannique a voulu faire croire, Les Aventures de John Davys ne sont donc pas « à moitié » copiées de cette publication. L'anecdote du régiment de Frohberg, largement revue et modifiée, ne prend pas tout à fait trois pages sur les 98 pages du roman dans l'édition Le Vasseur. Si on veut bien nous permettre un anachronisme, ce jugement - repris depuis sans vérification par tant de critiques qui croyaient s'élever en rabaissant Dumas - est un cas caractérisé de fake news avant la lettre. Et ce roman, sans avoir droit à la place d'honneur dans la vaste création du romancier, demeure un ouvrage très digne, marqué à chaque page par l'esthétique et l'éthique distinctives qui caractérisent l'ensemble de l'œuvre multiforme d'Alexandre Dumas.

Romans de Frederick Marryat consultés pour cette étude :

Pierre Simple, Gosselin, Paris, 1834.

Rattlin le marin, Gosselin, Paris, 1837.

Frank Mildmay, ou l'officier de la marine royale, Gosselin, Paris, 1837. Ardent Troughton, ou le commerçant naufragé, Gosselin, Paris, 1838.

M. Le Midshipman Aisé, Meline, Cans et Compagnie, Bruxelles, 1838. Le Vaisseau fantôme, Gosselin, Paris, 1839.

Joseph Rushbrook, ou le braconnier. Gosselin, Paris, 1841.

Le Pauvre Jack. Gosselin, Paris, 1841.

Le Pacha à mille et une queues, Gosselin, Paris, 1844.

Japhet, ou à la recherche d'un père, Gosselin, Paris, 1845.

Le Pirate et les trois cutters, Gosselin, Paris, 1845.

M. Violette, ou aventures d'un jeune émigré français de 1830, Gosselin, Paris, 1845.

Newton Forster, ou la marine marchande, Gosselin, Paris, 1846. 